\title{
Absolute Units and Electrical Measurements
}

\author{
By Sir Richard Glazebrook, K.C.B., F.R.S.
}

$\mathrm{T}$ $\mathrm{HE}$ absolute system of electromagnetic units was developed by the British Association Committee on "Practical Standards for Electrical Measurements". Of this Committee Sir Wm. Thomson (Lord Kelvin) and Clerk Maxwell were the leading members and the development of the system is mainly due to them. It may be of interest to have here a brief account of what is meant by an 'absolute system'.

The meaning given to the term 'A bsolute Measurement' was clearly explained by the B.A. Committee in the report to the Newcastle meeting in 1863. The Committee states:-

"The word 'absolute' in the present sense is used as opposed to the word 'relative' and by no means implies that the measurement is accurately made or that the unit employed is of perfect construction, but only that the measurement, instead of being a simple comparison with an arbitrary quantity of the same kind as that measured, is made by reference to certain fundamental units of another kind treated as postulates."

"For true absolute measurement the unit of force is defined as the force capable of producing the unit of velocity in the unit of mass when it has acted on it for the unit of time. Hence this force acting through the unit of space performs the absolute unit of work. In these two definitions, time, mass, and space are alone involved and the units in wbich these are measured, i.e., the second, gramme and metre* will alone in what follows be considered as fundamental units."

The Report then goes on to consider the mechanical, chemical and thermal effects due to electrical action, and since the chemical and thermal effects are measured by reference to a mechanical unit of work, concludes that it is only necessary in developing an absolute system of measurement to attend to the connexion between electrical magnitudes and the mechanical units. We are thus led to the following explanation of an absolute system of measurement.

Electric or magnetic systems exert force and are sources of energy. Force and energy are measured in terms of length, mass and time.

The forces between two quantities of electricity or two magnetic poles, measured in any units whatever, can be shown to be proportional to the pro-

* The metre was changed later to the centimetre. duct of the charges or of the poles and inversely to the squares of the distance between them. The constants of this proportion form the links required to connect quantities of electricity or of magnetism with the fundamental units. A third constant is required to determine the relation between a quantity of magnetism and an electric current. These three constants, usually denoted by $K_{0}, \mu_{0}$ and $A$, are reduced to two because it can be shown that

$$
A^{2} / \mu_{0} K_{0}=C^{2},
$$

where $C$ is the velocity of wave propagation.

In order to form an absolute system of electrical measurement, it is absolutely necessary to know two of these constants, which thus form the links connecting electrical and magnetic quantities with the fundamental units of length, mass and time. Such is the basis of the system of measurement conceived by Maxwell and the B.A. Committee in the year 1863 .

The committee selected as fundamental units the centimetre, the gram and the second, thus establishing the C.G.S. system. That, of course, was not necessary; the metre, the kilogram and the second might have been taken, but the choice was deliberate and only arrived at after much inquiry*.

To complete the electromagnetic system the Committee had to select the values of the two constants $\mu_{0}$ and $A$. It was at liberty to select any values it pleased for these; the selection would determine the units in terms of which quantities of magnetism and currents of electricity were to be measured. Further investigation showed that $\mu_{0} / A$ represents permeability-the ratio of the magnetic induction at a point in space to the magnetising force at that point-and it was a very natural step to assume that this ratio was unity, that is, to select the permeability of space as the unit of permeability, and thus to make $\mu_{0} / A=1$ on the C.G.S. system of absolute units.

A further assumption was required as to $A$, and for this the Committee selected the value unity. It thus formed the C.G.S. absolute system of electrical measurement with the centimetre, gram and second as basis, together with unity as the value of each of the necessary constants $\mu_{0}$ and $A$. * See B.A. Reports on Electrical Measurements : A Record of the
History of "Absolute Units" and of Lord Kelvin's work in connection History of "Absolute Units" and of Lord Kelvin's work in connection
with these. Reprinted by the Association, 1912 (Cambridge University with these. Reprinted by the Association, 1912 (Cambridge University
Press). See also "Dictionary of Applied Physics", vol. 2, page 941, Press). See also "Dictionary of Applied 
The last assumption, that $A$ is unity, implies that $\mu_{0} K_{0} C^{2}$ is unity, and hence that $K_{0}=1 / \mu_{0} C^{2}$.

For mechanical purposes the M.K.S. system is excellent and has many advantages, the main one perhaps being that the Joule, $10^{7}$ C.G.S. units of energy, is on that system unity. But in my view the M.K.S. (Resistance) system is not an absolute system of electrical measurement. A material substance-a tube of mercury or a coil of wire-will not of itself serve to connect electrical quantities with length, mass and time. There is a sense perhaps in which the M.K.S. $\Omega$ system ( $\Omega$ the ohm) may be looked on as a kind of hybrid absolute system because the ohm, $10^{9}$ absolute C.G.S. units of resistance as defined by the B.A. Committee, is by that system linked to the units of length, mass and time. Personally I should greatly regret to see the word 'absolute' divorced from its original meaning.

The M.K.S. system can, of course, become 'absolute' for electrical measurements just as the C.G.S. system has done. Three constants will be required : we may call them $K_{1}, \mu_{1}$ and $A_{1}$. They will be connected by the equation

$$
A_{1}^{2} / \mu_{1} K_{1}=C_{1}^{2} \text {, }
$$

where $C_{1}$ is measured in metres per second.

On the C.G.S. system, $A$ is an undimensional constant arbitrarily assumed equal to unity; we may agree to make the similar assumption on the M.K.S. system and thus put $A_{1}=$ unity. We are also free to assume any value we like for $\mu_{1}$ which leads to the result that $K_{1}=\left(\mu_{1} C_{1}{ }^{2}\right)^{-1}$, and we have then a second quite satisfactory system of 'absolute measurement'. This will usually differ from the C.G.S. system. If, however, we choose for $\mu_{1}$ the value $10^{-7}$, the M.K.S. system leads to practical results identical with those of the C.G.S. system, and the accompanying table gives the relations between the various quantities involved-it might, of course, be extended to include others.
M.K.S. system, may be selected as a fourth fundamental quantity required by the M.K.S. system. It must, of course, be remembered that a fifth such quantity is needed and completeness is attained by assuming for the value of $A_{1}$ unity, as the B.A. Committee did for $A$.

At a conference at which I was present recently the question was asked: "What is the need for a 'fourth unit'?" The reply was to the effect that the 'fourth unit' is needed to complete the dimen. sional equations. Thus it is not put forward to simplify electromagnetic theory or place it on a firmer basis, but to fill a supposed gap in the dimensional equations.

Dimensions, as the term is used by Maxwell, have reference to the three fundamental quantities of length, mass and time, and he set out with the object of expressing all electrical cuantities in terms of these three. This proved to be impossible. Using more modern terms and symbois, the quantities denoted now by $A, \mu_{0}$ and $K_{0}$ are involved and these are connected as explained above by the equation $A^{2} / \mu_{0} K_{0}=C^{2}$.

The dimensions of $\mu_{0} K_{0} / A^{2}$ are those of the reciprocal of the square of a velocity, but without some further assumptions we can proceed no further. Maxwell tacitly assumed $A$ to be unity and undimensional, leading to the result that $\mu_{0} K_{0}=1 / C^{2}$

Maxwell points out that two assumptions are possible: on the one, the electromagnetic, $\mu_{0}$ is treated as an undimensional constant of value unity and we have the electromagnetic system, on which, for example, the dimensions of a resistance are those of a velocity, those of a coefficient of induction are a length, etc. For the electrostatic system, $K_{0}$ is treated as an undimensional constant of value unity.

There is, however, another solution to the equation $\mu_{0} K_{0}=1 / C^{2}$. We may assume $\mu_{0}$ and $K_{0}$ each to have unknown dimensions in length, mass

Table Showing the Redation Between Quantities on the C.G.S. Electromagnetic System AND ON THE PROPOSED M.K.S. SYSTEM

\begin{tabular}{|c|l|l|c|c|c|c|c|c|}
\hline & Iength & Mass & Time & $\begin{array}{c}\text { Space } \\
\text { Permeability } \\
\mu_{0} / \boldsymbol{A}\end{array}$ & $\begin{array}{c}\text { Electromagnetic } \\
\text { Coefficient A }\end{array}$ & Coulomb & Ampere & Volt \\
\hline C.G.S. & $1 \mathrm{~cm}$. & 1 gramme & 1 second & 1 & 1 & $10^{-1}$ & $10^{-1}$ & $10^{8}$ \\
\hline M.K.S. & 1 metre & 1 kilogramme & 1 second & $10^{-7}$ & 1 & 1 \\
\hline
\end{tabular}

On Maxwell's theory of the electrical field the permeability of space is assumed to be a quantity having dimensions. The value, therefore, will depend on the system of units employed. The symbol $\mu_{0}$ is used to denote this quantity. On the C.G.S. system the permeability of space has been selected as the unit of permeability. Hence on this system the value of $\mu_{0}$ is unity. On the M.K.S. system its value is $10^{-7}$, and on the 'rationalized' M.K.S. system the value of $\mu_{0}$ is $4 \pi \times 10^{-7}$.

At the same time the S.U.N. Commission desires to take this opportunity of placing on record its recognition of the fact that there are important electrical theories supported by a number of physicists in accordance with which $\mu_{0} K$ is a pure number.

This, then, briefly gives my reason for the desire that $10^{-7}$, the value of space permeability* on the

* In cases in which it is desired to use the "rationalized system of
units", this will become $4 \pi \times 10^{-7}$, while the value of $A$ will be unity. and time, but to be so connected that the dimensions of their product are those of $1 / C^{2}$. The dimensions of a coefficient of induction are then those of $\mu_{0} \times L$; those of a resistance are those of 
$\mu_{0} L / T$, and both are unknown. But nothing is really gained by taking either of these quantities as a 'fourth unit' and treating it either as a fourth dimension additional to those of length, mass and time, or as a quantity of unknown dimensions in length, mass and time.

The first alternative is an additional complication to electromagnetic theory-it does no doubt simplify certain dimensional equations ; the second is secured by the acceptance by the I.E.C. of the statement that ' $\mu_{0}$ possesses physical dimensions'.
What is necessary to place the M.K.S. system on a secure basis is the realisation of the facts that the two quantities $A$ and $\mu_{0}$ are essential, and that if the units of the system are to be the practical units of the C.G.S. system, their values (unrationalised) must be $I$ and $10^{-7}$ and (rationalised) $I$ and $4 \pi \times 10^{-7}$.

It must, of course, be recognised that another solution to the equation $A^{2} / \mu_{0} K_{0}=C^{2}$ is open to us. We may, with Gauss, put $A=C$ and then $\mu_{0} K_{0}$ becomes a numeric.

\title{
Adolf von Baeyer, I835-I9I7
}

\author{
By Prof. J. R. Partington, M.B.E.
}

$\mathrm{T}$ WO of the outstanding organic chemists of the later nineteenth and early twentieth centuries were Adolf von Baeyer and his pupil Emil Fischer. As the late Prof. W. H. Perkin said, "their influence has been profound, mainly no doubt because of the immense amount of work of fundamental importance which they have left behind, but to a scarcely less degree by reason of their influence as teachers". The discoveries of Baeyer have also had a most important bearing on chemical industry, although he himself was little interested in their commercial exploitation. The side of theoretical organic chemistry which interested him most was the structural aspect, and in this his views, even in his early publications, were remarkably accurate. He modified in many ways the picture as left by Kekulé, and in some parts modern developments have merely filled in the details.

Adolf von Baeyer was born in Berlin on October 31,1835 . His chemical training began, like that of so many others eminent in this science, with Bunsen at Heidelberg. There he worked on cacodyl compounds, already made important in the early history of organic chemistry by the investigations of Bunsen. The latter had little interest in the development of organic chemistry at that time, and Baeyer, who wished to take up this branch of the science, spent two years in a private laboratory of Kekulé at Heidelberg. In 1858 he followed Kekule to Ghent and began work on derivatives of uric acid. His interest in this was aroused in a curious way. During his journey from Heidelberg he became acquainted with a former pupil of Liebig, who gave him a box of specimens of uric acid derivatives. The further investigation of this class of bodies, carried out by Baeyer and his pupil Emil Fischer, may there- fore be said to go back directly to the early work of Liebig at Giessen.

In 1860 Baeyer returned to Berlin as a teacher of organic chemistry in a small technical school. In this poorly paid and modest position he remained for twelve years, during which period he had in his laboratory such men as Graebe, Liebermann and Victor Meyer. The work on uric acid derivatives was continued, and in this period Baeyer also put forward the suggestion (in 1870) that the formation of sugar in plants is due to the reduction of carbonic acid, HO.CO.OH, in the green leaves and under the influence of light, to formaldehyde, $\mathrm{H} . \mathrm{COH}$, which then undergoes poly. merisation to grape sugar, $\mathrm{C}_{6} \mathrm{H}_{12} \mathrm{O}_{6}$. The study of a very important class of reactions called condensations began in 1866 with an investigation of the action of acids on acetone, and was developed in various directions, including the condensation of hydrocarbons with phenols and aldehydes. One outcome of this work was the use of phthalic anhydride, which led to the discovery of the phthaleins, many of which are dyestuffs, such as fluorescein, eosin and the rhodamines.

After a period from 1870 until 1875 at Strassburg, where Emil Fischer was his pupil, Baeyer succeeded Liebig at Munich. For more than twenty years, Liebig's activities at Munich had been confined to lecturing and studying the applications of chemistry to agriculture and medicine. There was no laboratory, and Baeyer's first task was to plan large new laboratories which were completed in 1877. In these he continued the investigation of indigo which he had begun in 1865, preparing several derivatives. He found that isatin (IV), a red substance discovered by Laurent in 1840 and produced by oxidising indigo with nitric acid, on reduction yields first dioxindole (III) and then 